\title{
Preparation and Synthetic Application of Novel Ketene Silyl Acetal of Methyl Trifluoropyruvate
}

\author{
Go Takikawa, Toshimasa Katagiri and Kenji Uneyama* \\ Department of Applied Chemistry, Faculty of Engineering, Okayama University \\ 3-1-1 Tsushima-naka, Okayama 700-8530, Japan \\ uneyamak@,cc.okayama-u.ac.jp
}

General Procedures

3,3,3-Trifluoro-2-[(4-methoxyphenyl)(trimethylsilanyloxy)methyl]-2-

trimethylsilanyloxypropanoic acid methyl ester (10a) ....................... S2

3,3,3-Trifluoro-2-[(3-chlorophenyl)(trimethylsilanyloxy)methyl]-2-

trimethylsilanyloxypropanoic acid methyl ester (10b). ..................... S2

3,3,3-Trifluoro-2-[(4-trifluoromethylphenyl)(trimethylsilanyloxy)methyl]-2-

trimethylsilanyloxypropanoic acid methyl ester (10d). ...................... S3

3,3,3-Trifluoro-2-[furyl(trimethylsilanyloxy)methyl]-2-trimethylsilanyloxypropanoic acid methyl ester (10e). ......................... S3

3,3,3-Trifluoro-2-[(4-methylphenyl)(trimethylsilanyloxy)methyl]-2-

trimethylsilanyloxypropanoic acid methyl ester (10f) ...................... S4

3,3,3-Trifluoro-2-hydroxy-2-[(4-methoxyphenyl)hydroxymethyl]propanoic acid methyl ester (12a). .......................... S4

2-[(3-Chlorophenyl)hydroxymethyl]-3,3,3-trifluoro-2-hydroxypropanoic acid methyl ester (12b).

3,3,3-Trifluoro-2-hydroxy-2-[(4-trifluoromethylphenyl)hydroxymethyl]propanoic acid methyl ester (12d). ......................... S5

3,3,3-Trifluoro-2-[(furyl)hydroxymethyl]-2-hydroxypropanoic acid methyl ester (12e). ............................ S5

3,3,3-Trifluoro-2-hydroxy-2-[1'-(p-tolyl)hydroxymethyl]propanoic acid methyl ester (12f). ............................. S6

2-Trifluoromethyl-2,3-dihydroxy-5-phenylpentanoic acid methyl ester (12g)...... S6

2-Trifluoromethyl-2,3-dihydroxy-4-hexenoic acid methyl ester (12h). ........... S6

2-Hydroxy-3-oxo-2-trifluoromethyloctanoic acid methyl ester (15b). ............ S7 


\section{General Procedures}

${ }^{1} \mathrm{H}$ NMR spectra were recorded at 300, 500 and $600 \mathrm{MHz},{ }^{19} \mathrm{~F}$ NMR was recorded at 282 and 470 $\mathrm{MHz}$ and ${ }^{13} \mathrm{C} \mathrm{NMR}$ was recorded at $151 \mathrm{MHz}$. The chemical shifts are reported in $\delta(\mathrm{ppm})$ values

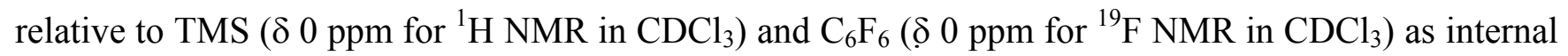
standards, respectively. Coupling constants are reported in hertz (Hz). All air and/or water-sensitive reactions were carried out under an argon atmosphere with dry, freshly distilled solvents using a standard syringe-cannula/septa technique. MTFP (1, 98\% pure), was distilled from $\mathrm{P}_{2} \mathrm{O}_{5}$. THF was distilled from sodium/benzophenone ketyl. $n$-Heptane and TMSCl were distilled from $\mathrm{CaH}_{2} \cdot \mathrm{CH}_{2} \mathrm{Cl}_{2}$ was distilled from $\mathrm{P}_{2} \mathrm{O}_{5}$. Aldehydes, acyl halides, ketones and benzaldehyde dimethyl acetal were purified by distillation. Other reagents were employed without further purification. Kanto Chemical Co., Inc. silica gel (Silica Gel 60N (spherical, neutral), 40-50 $\mu \mathrm{m}$ ) was employed for the chromatography.

\section{3,3,3-Trifluoro-2-[(4-methoxyphenyl)(trimethylsilanyloxy)methyl]-2-}

\section{trimethylsilanyloxypropanoic acid methyl ester (10a).}

97\%; colorless oil; Diastereomeric mixture (63:37 by ${ }^{19}$ F NMR); IR (neat) 2990, 2940, 2880, 1785 , 1766, 1632, 1602, 1528, 1528, 1480, $1458 \mathrm{~cm}^{-1} ;{ }^{1} \mathrm{H}$ NMR (300 MHz) $\delta-0.01$ (s, 9H, major), 0.07 (s, $9 \mathrm{H}$, minor), 0.06 (s, 9H, major), 0.21 (s, 9H, minor), 3.71 (s, 3H, major), 3.80 (s, 3H, major), 3.81 (s, $3 \mathrm{H}$, minor), 3.86 (s, 3H, minor), $5.18(\mathrm{~s}, 1 \mathrm{H}$, minor), 5.20 (s, 1H, major), $6.80(\mathrm{dd}, J=1.8 \mathrm{~Hz}, J=6.9 \mathrm{~Hz}$, 2H, major), 6.82 (d, $J=8.7 \mathrm{~Hz}, 2 \mathrm{H}$, minor), 7.19 (dd, $J=1.8 \mathrm{~Hz}, J=6.9 \mathrm{~Hz}, 2 \mathrm{H}$, major), 7.27 (d, $J=$ $8.7 \mathrm{~Hz}, 2 \mathrm{H}) ;{ }^{19} \mathrm{~F}$ NMR $(282 \mathrm{MHz}) \delta 87.6$ (s, 3F, minor), 89.9 (s, 3F, major); EI MS m/z (relative intensity) $423\left(\mathrm{M}^{+}-15\right.$, trace), 209 (100), 73 (44); Anal. Calcd for $\mathrm{C}_{18} \mathrm{H}_{29} \mathrm{~F}_{3} \mathrm{O}_{5} \mathrm{Si}_{2}$ : C, 49.29; H, 6.66. Found; C, 49.46; H, 6.83.

\section{3,3,3-Trifluoro-2-[(3-chlorophenyl)(trimethylsilanyloxy)methyl]-2-trimethylsilanyloxypropanoic acid methyl ester (10b).}


$86 \%$; colorless oil; Diastereomeric mixture (61:39 by ${ }^{19}$ F NMR); IR (neat) 2964, 2910, 1772, 1760 , 1600, 1580, 1478, $1440 \mathrm{~cm}^{-1} ;{ }^{1} \mathrm{H}$ NMR (300 MHz) $\delta-0.04$ (s, 9H, minor), 0.02 (s, 9H, major), 0.06 (s, 9H, major), $0.20(\mathrm{~s}, 9 \mathrm{H}$, minor), $3.73(\mathrm{~s}, 3 \mathrm{H}$, major), $3.86(\mathrm{~s}, 3 \mathrm{H}$, minor), 5.18 (s, 1H, minor), 5.23 (s, $1 \mathrm{H}$, major), 7.08-7.39 (m, 5H x 2, major and minor mixed); ${ }^{19} \mathrm{~F}$ NMR (282 MHz) $\delta 87.7$ (s, 3F, minor), 89.9 (s, 3F, major); EI MS m/z (relative intensity) 427 ( $\mathrm{M}^{+}-15$, trace), 302 (1), 213 (100), 73 (80); Anal. Calcd for $\mathrm{C}_{17} \mathrm{H}_{26} \mathrm{ClF}_{3} \mathrm{O}_{4} \mathrm{Si}_{2}$ : C, 46.09; H, 5.92. Found; C, 45.79; H, 6.16.

\section{3,3,3-Trifluoro-2-[(4-trifluoromethylphenyl)(trimethylsilanyloxy)methyl]-2- trimethylsilanyloxypropanoic acid methyl ester (10d).}

58\%; colorless oil; Diastereomeric mixture (53:47 by ${ }^{19}$ F NMR); IR (neat) 3000, 2948, 2897, 1958, 1783, 1635, 1458, $1437 \mathrm{~cm}^{-1} ;{ }^{1} \mathrm{H}$ NMR (500 MHz) $\delta-0.06$ (s, 9H, minor), 0.00 (s, 9H, major), 0.03 (s, 9H, major), 0.17 (s, 9H, minor), 3.74 (s, 3H, major), 3.87 (s, 3H, minor), 5.27 (s, 1H, minor), 5.30 (s, $1 \mathrm{H}$, major), $7.41(\mathrm{~d}, J=8.5 \mathrm{~Hz}, 1 \mathrm{H} \mathrm{x} \mathrm{2,} \mathrm{major} \mathrm{and} \mathrm{minor} \mathrm{mixed),} 7.47$ (d, $J=8.0 \mathrm{~Hz}, 1 \mathrm{H} \mathrm{x} \mathrm{2,} \mathrm{major} \mathrm{and}$ minor mixed), 7.55 (d, $J=8.5 \mathrm{~Hz}, 1 \mathrm{H} \mathrm{x} 2$, major and minor mixed), 7.56 (d, $J=8.0 \mathrm{~Hz}, 1 \mathrm{H} \times 2$, major and minor mixed); ${ }^{19} \mathrm{~F}$ NMR (470 MHz) $\delta 88.4$ (s, 3F, minor), 90.8 (s, 3F, major), 99.2 (s, 3F, major), 99.3 (s, 3F, minor); EI MS m/z (relative intensity) $461\left(\mathrm{M}^{+}-15,2\right), 302$ (5), 247 (100), 73 (55); Anal. Calcd for $\mathrm{C}_{18} \mathrm{H}_{26} \mathrm{~F}_{6} \mathrm{O}_{4} \mathrm{Si}_{2}$ : C, 45.37; H, 5.50. Found; C, 45.25; H, 5.44.

\section{3,3,3-Trifluoro-2-[furyl(trimethylsilanyloxy)methyl]-2-trimethylsilanyloxypropanoic} methyl ester (10e).

50\%; colorless oil; Diastereomeric mixture (64:36 by GC analysis); IR (neat) 2968, 2908, 1772, 1758, $1440 \mathrm{~cm}^{-1} ;{ }^{1} \mathrm{H}$ NMR (600 MHz) $\delta-0.03(\mathrm{~s}, 9 \mathrm{H}$, major), 0.05 (s, 9H, minor), $0.11(\mathrm{~s}, 9 \mathrm{H}$, minor), 0.21 (s, 9H, major), 3.76 (s, 3H, minor), 3.84 (s, 3H, major), 5.28 (s, 1H, major), 5.33 (s, 1H, minor), 6.29-6.32 (m, 2H, minor), $6.35(\mathrm{dd}, J=1.8 \mathrm{~Hz}, J=3.0 \mathrm{~Hz}, 1 \mathrm{H}$, major), $6.36(\mathrm{~d}, J=3.0 \mathrm{~Hz}, 1 \mathrm{H}$, major), 7.31 (dd, $J$ $=0.6 \mathrm{~Hz}, J=1.8 \mathrm{~Hz}, 1 \mathrm{H}$, minor), $7.37\left(\mathrm{dd}, J=0.6 \mathrm{~Hz}, J=1.8 \mathrm{~Hz}, 1 \mathrm{H}\right.$, major); ${ }^{19} \mathrm{~F}$ NMR $(282 \mathrm{MHz}) \delta$ 
86.4 (s, 3F, major), 89.2 (s, 3F, minor); EI MS m/z (relative intensity) $383\left(\mathrm{M}^{+}-15,2\right), 302$ (trace), 169 (100), 73 (59); Anal. Calcd for $\mathrm{C}_{15} \mathrm{H}_{25} \mathrm{~F}_{3} \mathrm{O}_{5} \mathrm{Si}_{2}$ : C, 45.21; H, 6.32. Found; C, 44.91; H, 6.33.

\section{3,3,3-Trifluoro-2-[(4-methylphenyl)(trimethylsilanyloxy)methyl]-2- trimethylsilanyloxypropanoic acid methyl ester (10f).}

20\%; colorless oil; Diastereomeric mixture (86:14 by ${ }^{19}$ F NMR); IR (neat) 2980, 2950, 2928, 1780 , $1638,1526,1450 \mathrm{~cm}^{-1} ;{ }^{1} \mathrm{H}$ NMR $(300 \mathrm{MHz}) \delta-0.07$ (s, 9H, major), 0.00 (s, 9H, minor), 0.05 (s, 9H, minor), $0.20(\mathrm{~s}, 9 \mathrm{H}$, major), $2.32(\mathrm{~s}, 3 \mathrm{H}$, minor), 2.35 (s, 3H, major), 3.72 (s, 3H, minor), 3.86 (s, $3 \mathrm{H}$, major), 5.19 (s, 1H, major), $5.22(\mathrm{~s}, 1 \mathrm{H}$, minor), 7.05 (d, J=8.1 Hz, 2H, minor), 7.09 (d, J=8.1 Hz, 2H, major), 7.15 (d, $J=8.1 \mathrm{~Hz}, 2 \mathrm{H}$, minor), $7.23\left(\mathrm{~d}, J=8.1 \mathrm{~Hz}, 2 \mathrm{H}\right.$, major); ${ }^{19} \mathrm{~F}$ NMR (282 MHz) $\delta 87.6$ (s, 3F, major), 89.8 (s, 3F, minor); EI MS m/z (relative intensity) 405 ( $\left.\mathrm{M}^{+}-17,2\right), 193$ (100), 73 (32); Anal. Calcd for $\mathrm{C}_{18} \mathrm{H}_{29} \mathrm{~F}_{3} \mathrm{O}_{4} \mathrm{Si}_{2}$ : C, 51.16; H, 6.92. Found; C, 50.95; H, 6.97.

\section{3,3,3-Trifluoro-2-hydroxy-2-[(4-methoxyphenyl)hydroxymethyl]propanoic acid methyl ester} (12a).

87\%; oily solid; Diastereomeric mixture (52:48 by ${ }^{1} \mathrm{H}$ NMR); mp $48-49{ }^{\circ} \mathrm{C}$; IR (neat) 3512,3016 , $2968,2848,1754,1766,1616,1588,1516,1444,1480,1458 \mathrm{~cm}^{-1} ;{ }^{1} \mathrm{H}$ NMR $(600 \mathrm{MHz}) \delta 2.63(\mathrm{~s}, J=$ $7.8 \mathrm{~Hz}, 1 \mathrm{H}$, minor), 2.91 (d, $J=7.2 \mathrm{~Hz}, 1 \mathrm{H}$, major), 3.70 (s, 3H, major), 3.78 (s, 3H, major), 3.80 (s, $3 \mathrm{H}$, minor), 3.97 (s, 3H, minor), 3.99 (s, 1H, major), 4.18 (s, 1H, minor), 5.15 (d, J= $7.2 \mathrm{~Hz}, 1 \mathrm{H}$, minor), $5.20(\mathrm{~s}, J=7.8 \mathrm{~Hz}, 1 \mathrm{H}$, major), 6.84 (dd, $J=1.8 \mathrm{~Hz}, J=6.6 \mathrm{~Hz}, 2 \mathrm{H}$, major), 6.88 (dd, $J=1.8 \mathrm{~Hz}, J=$ $6.6 \mathrm{~Hz}, 2 \mathrm{H}$, minor), 7.24 (dd, $J=1.8 \mathrm{~Hz}, J=6.6 \mathrm{~Hz}, 2 \mathrm{H}$, major), 7.38 (d, $J=1.8 \mathrm{~Hz}, J=6.6 \mathrm{~Hz}, 2 \mathrm{H}$, minor); ${ }^{19} \mathrm{~F}$ NMR (282 MHz) $\delta 87.81$ (s, 3F, minor), 87.82 (s, 3F, major); EI MS m/z (relative intensity) $294\left(\mathrm{M}^{+}\right.$, trace), 137 (100), 109 (33), 94 (23); 77 (22); Anal. Calcd for $\mathrm{C}_{12} \mathrm{H}_{13} \mathrm{~F}_{3} \mathrm{O}_{5}$ : C, 48.99; H, 4.45. Found; C, 48.97; H, 4.41. 
2-[(3-Chlorophenyl)hydroxymethyl]-3,3,3-trifluoro-2-hydroxypropanoic acid methyl ester (12b).

79\%; white powder; Diastereomeric mixture (64:36 by ${ }^{1} \mathrm{H} \mathrm{NMR}$ ); mp $106-108{ }^{\circ} \mathrm{C}$; IR (neat) 3508 , 2965, 1754, 1760, 1600, 15780, 1480, $1442 \mathrm{~cm}^{-1} ;{ }^{1} \mathrm{H}$ NMR $(300 \mathrm{MHz}) \delta 2.71(\mathrm{~d}, J=7.8 \mathrm{~Hz}, 1 \mathrm{H}$, minor), $3.00(\mathrm{~d}, J=8.1 \mathrm{~Hz}, 1 \mathrm{H}$, major), 3.75 (s, 3H, major), 3.98 (s, 1H, major), 3.99 (s, 3H, minor), 4.19 (s, 1H, minor), 5.17 (d, $J=7.2 \mathrm{~Hz}, 1 \mathrm{H}$, minor), 5.23 (d, $J=7.8 \mathrm{~Hz}, 1 \mathrm{H}$, major), 7.18-7.48 (m, $4 \mathrm{H} \mathrm{x}$ 2, major and minor mixed); ${ }^{19} \mathrm{~F}$ NMR (282 MHz) $\delta 87.88$ (s, 3F, major), 87.90 (s, 3F, minor); EI MS m/z (relative intensity) $298\left(\mathrm{M}^{+}\right.$, trace), 141 (100), 113 (36), 77 (69); Anal. Calcd for $\mathrm{C}_{11} \mathrm{H}_{10} \mathrm{ClF}_{3} \mathrm{O}_{4}: \mathrm{C}_{\text {, }}$ 44.24; H, 3.38. Found; C, 44.04; H, 3.46.

\section{3,3,3-Trifluoro-2-hydroxy-2-[(4-trifluoromethylphenyl)hydroxymethyl]propanoic acid methyl} ester (12d).

$68 \%$; white powder; Diastereomeric mixture (67:33 by $\left.{ }^{1} \mathrm{H} \mathrm{NMR}\right)$; mp $108-110{ }^{\circ} \mathrm{C}$; IR (neat) 3525 , 3000, 1765, 1640, $1460 \mathrm{~cm}^{-1} ;{ }^{1} \mathrm{H}$ NMR $(500 \mathrm{MHz}) \delta 2.63(\mathrm{~d}, J=7.8 \mathrm{~Hz}, 1 \mathrm{H}$, minor), $2.93(\mathrm{~d}, J=8.1 \mathrm{~Hz}$, 1H, major), 3.75 (s, 3H, major), 3.96 (s, 1H, major), 4.01 (s, 3H, minor), 4.19 (s, 1H, minor), 5.27 (d, J $=7.8 \mathrm{~Hz}, 1 \mathrm{H}$, minor), $5.33(\mathrm{~d}, J=8.1 \mathrm{~Hz}, 1 \mathrm{H}$, major), 7.45-7.66 (m, $4 \mathrm{H} \mathrm{x} \mathrm{2,} \mathrm{major} \mathrm{and} \mathrm{minor} \mathrm{mixed);}$

${ }^{19} \mathrm{~F}$ NMR (470 MHz) $\delta 87.88(\mathrm{~s}, 3 \mathrm{~F}$, minor), 87.90 (s, 3F, major), 98.94 (s, 3F, major), 98.95 (s, 3F, minor); EI MS m/z (relative intensity) $313\left(\mathrm{M}^{+}-19,4\right), 175$ (100), 158 (45), 127 (80); Anal. Calcd for $\mathrm{C}_{12} \mathrm{H}_{10} \mathrm{~F}_{6} \mathrm{O}_{4}: \mathrm{C}, 43.39 ; \mathrm{H}, 3.03$. Found; $\mathrm{C}, 43.39 ; \mathrm{H}, 3.25$.

\section{3,3,3-Trifluoro-2-[(furyl)hydroxymethyl]-2-hydroxypropanoic acid methyl ester (12e).}

73\%; brown oil; Diastereomeric mixture (63:37 by ${ }^{19} \mathrm{~F}$ NMR); IR (neat) 3504, 2968, 1756, 1504, $1444 \mathrm{~cm}^{-1} ;{ }^{1} \mathrm{H}$ NMR (300 MHz) $\delta 3.04$ (br, $1 \mathrm{H} \mathrm{x} \mathrm{2,} \mathrm{major} \mathrm{and} \mathrm{minor} \mathrm{mixed),} 3.81$ (s, 3H, minor), 3.98 (s, 3H, major), 4.07 (s, 1H, minor), 4.27 (s, 1H, major), 5.23 (br, 1H, major), 5.30 (br, 1H, minor), 6.32$6.35(\mathrm{~m}, 2 \mathrm{H}$ x 2, major and minor mixed), $6.37(\mathrm{dd}, J=1.8 \mathrm{~Hz}, J=3.3 \mathrm{~Hz}, 2 \mathrm{H}$, major), $6.46(\mathrm{dd}, J=0.3$ $\mathrm{Hz}, J=3.3 \mathrm{~Hz}, 1 \mathrm{H}$, major), 7.35 (dd, $J=0.9 \mathrm{~Hz}, J=1.8 \mathrm{~Hz}, 1 \mathrm{H}$, minor), 7.31 (dd, $J=0.9 \mathrm{~Hz}, J=1.8$ $\mathrm{Hz}, 1 \mathrm{H}$, minor); ${ }^{19} \mathrm{~F}$ NMR $(282 \mathrm{MHz}) \delta 86.9$ (s, 3F, major), 87.8 (s, 3F, minor); EI MS m/z (relative 
intensity) $254\left(\mathrm{M}^{+}\right.$, trace), 177 (4), 97 (100), 69 (13); Anal. Calcd for $\mathrm{C}_{9} \mathrm{H}_{9} \mathrm{~F}_{3} \mathrm{O}_{5}: \mathrm{C}, 42.53$; H, 3.57.

Found; C, 42.72; H, 3.68 .

\section{3,3,3-Trifluoro-2-hydroxy-2-[1'-(p-tolyl)hydroxymethyl]propanoic acid methyl ester (12f).}

88\%; white powder; Diastereomeric mixture (65:35 by ${ }^{1} \mathrm{H}$ NMR); mp $80-82{ }^{\circ} \mathrm{C}$; IR (neat) 3525,3070 , 2990, 1755, 1525, $1458 \mathrm{~cm}^{-1} ;{ }^{1} \mathrm{H}$ NMR (300 MHz) $\delta 2.33$ (s, 3H, major), 2.35 (s, 3H, minor), 2.47 (d, J $=7.5 \mathrm{~Hz}, 1 \mathrm{H}$, minor), 2.77 (d, J = 8.4 Hz, 1H, major), 3.72 (s, 3H, major), 3.90 (s, 1H, major), 4.00 (s, $3 \mathrm{H}$, minor), 4.12 (s, 1H, minor), 5.17 (d, $J=7.2 \mathrm{~Hz}, 1 \mathrm{H}$, minor), 5.23 (d, $J=8.1 \mathrm{~Hz}, 1 \mathrm{H}$, major), 7.12$7.36\left(\mathrm{~m}, 4 \mathrm{H} \times 2\right.$, major and minor mixed); ${ }^{19} \mathrm{~F}$ NMR (282 MHz) $\delta 87.80$ (s, 3F, minor), 87.83 (s, 3F, major); EI MS m/z (relative intensity) $200\left(\mathrm{M}^{+}-78,11\right), 120$ (100), 92 (32) 77 (14); Anal. Calcd for $\mathrm{C}_{12} \mathrm{H}_{13} \mathrm{~F}_{3} \mathrm{O}_{4}$ : C, 51.80; H, 4.71. Found; C, 51.70; H, 4.75.

\section{2-Trifluoromethyl-2,3-dihydroxy-5-phenylpentanoic acid methyl ester (12g).}

53\%; colorless oil; Diastereomeric mixture (58:42 by ${ }^{19} \mathrm{~F}$ NMR); mp $42-44{ }^{\circ} \mathrm{C}$; IR (neat) 3524,3032 , 2968, 2872, 1744, 1606, 1500, $1444 \mathrm{~cm}^{-1} ;{ }^{1} \mathrm{H}$ NMR (500 MHz) $\delta 1.49-1.57$ (m, 1H, major), 1.84 (d, $J=$ $11.5 \mathrm{~Hz}, 1 \mathrm{H}$, major), 1.85-1.93 (m, $1 \mathrm{H} \mathrm{x} \mathrm{2,} \mathrm{major} \mathrm{and} \mathrm{minor} \mathrm{mixed),} \mathrm{1.99-2.05} \mathrm{(m,} \mathrm{1H,} \mathrm{minor),} 2.06$ (d, $J=9.0 \mathrm{~Hz}, 1 \mathrm{H}$, minor), $2.70(\mathrm{~m}, 1 \mathrm{H} \mathrm{x} \mathrm{2,} \mathrm{major} \mathrm{and} \mathrm{minor} \mathrm{mixed),} 2.93(\mathrm{~m}, 1 \mathrm{H} \mathrm{x} \mathrm{2,} \mathrm{major} \mathrm{and} \mathrm{minor}$ mixed), 3.86 (s, 3H, minor), 3.94 (s, 3H, major), 3.95 (s, 1H, minor), 4.03 (s, 1H, major), $4.12(\mathrm{~m}, 1 \mathrm{H} \mathrm{x}$ 2, major and minor mixed), 7.16-7.32 (m, 5H x 2, major and minor mixed); ${ }^{19} \mathrm{~F}$ NMR (282 MHz) $\delta 87.9$ (s, 3F, minor), 88.1 (s, 3F, major); EI MS m/z (relative intensity) $292\left(\mathrm{M}^{+}, 4\right), 158$ (29), 117 (55), 91 (100); Anal. Calcd for $\mathrm{C}_{13} \mathrm{H}_{15} \mathrm{~F}_{3} \mathrm{O}_{4}$ : C, 53.43; H, 5.17. Found; C, 53.28; H, 5.04.

\section{2-Trifluoromethyl-2,3-dihydroxy-4-hexenoic acid methyl ester (12h).}

64\%; colorless oil; Diastereomeric mixture (58:42 by ${ }^{1} \mathrm{H}$ NMR); IR (neat) $3516,3000,2955,2898$, 1765, 1690, $1460 \mathrm{~cm}^{-1} ;{ }^{1} \mathrm{H}$ NMR (300 MHz) $\delta 1.71$ (ddd, $J=0.3 \mathrm{~Hz}, J=1.8 \mathrm{~Hz}, J=6.6 \mathrm{~Hz}, 2 \mathrm{H}$, minor), 1.75 (dd, $J=1.8 \mathrm{~Hz}, J=6.6 \mathrm{~Hz}, 2 \mathrm{H}$, major), 2.04 (d, $J=8.4 \mathrm{~Hz}, 1 \mathrm{H}$, major), 2.28 (d, $J=8.4 \mathrm{~Hz}, 1 \mathrm{H}$, 
minor), 3.87 (s, 3H, minor), 3.95 (s, 3H, major), 3.96 (s, 1H, minor), 4.03 (d, J = $0.3 \mathrm{~Hz}, 1 \mathrm{H}$, major), 4.55 (d, $J=8.4 \mathrm{~Hz}, 1 \mathrm{H}$, major), 4.58 (d, $J=8.4 \mathrm{~Hz}, 1 \mathrm{H}$, minor), 5.42-5.50 (m, 1H, minor), 5.59-5.67 (m, $1 \mathrm{H}$, major), 5.77-5.94 (m, $1 \mathrm{H} \times 2$, major and minor mixed); ${ }^{19} \mathrm{~F}$ NMR (282 MHz) $\delta 87.6$ (s, 3F, major), 88.8 (s, 3F, minor); EI MS m/z (relative intensity) $211\left(\mathrm{M}^{+}-17\right.$, trace), 138 (6), 103 (6), 71 (100), 69 (20); Anal. Calcd for $\mathrm{C}_{8} \mathrm{H}_{11} \mathrm{~F}_{3} \mathrm{O}_{4}$ : C, 42.11; H, 4.86. Found; C, 42.09; H, 4.61.

\section{2-Hydroxy-3-oxo-2-trifluoromethyloctanoic acid methyl ester (15b).}

$72 \%$; diastereomeric mixture (50:50 by ${ }^{19} \mathrm{~F}$ NMR); colorless oil; IR (neat) 3005, 2968, 2880, 1795, $1785,1480,1460 \mathrm{~cm}^{-1} ;{ }^{1} \mathrm{H}$ NMR $(300 \mathrm{MHz}) \delta 0.90(\mathrm{~m}, 3 \mathrm{H}), 1.34(\mathrm{ddd}, J=2.1 \mathrm{~Hz}, J=7.2 \mathrm{~Hz}, J=7.5$ $\mathrm{Hz}, 4 \mathrm{H}), 1.69$ (m, 2H), 2.49 (td, $J=1.5 \mathrm{~Hz}, J=7.5 \mathrm{~Hz}, 2 \mathrm{H}), 3.85(\mathrm{~s}, 3 \mathrm{H}), 5.47$ (q, $J=7.2 \mathrm{~Hz}, 1 \mathrm{H}) ;{ }^{19} \mathrm{~F}$ NMR (282 MHz) $\delta 88.1$ (s, 3F), 88.2 (s, 3F); EI MS m/z (relative intensity) $237\left(\mathrm{M}^{+}-19\right.$, trace), 200 (20), 99 (100), 70 (63), 55 (41); Anal. Calcd for $\mathrm{C}_{10} \mathrm{H}_{15} \mathrm{~F}_{3} \mathrm{O}_{4}$ : C, 46.88; H, 5.90. Found; C, 46.61; H, 5.84. 